\title{
Allgemeine und forensische Psychiatrie - Wer kümmert sich um junge Menschen mit psychotischen Störungen?
}

\author{
General Psychiatry and Forensic Psychiatry - Who Cares for Young People with \\ Psychotic Disorders?
}

Autor

Institut
Martin Zinkler

East London NHS Foundation Trust, Newham Centre for Mental Health, London

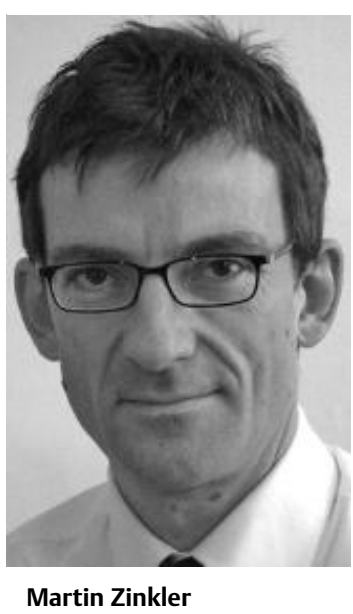

Bibliografie

DOI $10.1055 / \mathrm{s}-0028-1090168$

Psychiat Prax 2009; 36:

103-105

(c) Georg Thieme Verlag KG

Stuttgart $\cdot$ New York .

ISSN 0303-4259

Korrespondenzadresse

\section{Dr. Martin Zinkler}

Newham Centre for Mental Health, Cherry Tree Way, Glen Rd, GB-London E13 8SP martin.zinkler@eastlondon. nhs.uk
Der Landesbeauftragte für den Maßregelvollzug (MRV) in Nordrhein-Westfalen hat eine Arbeitsgruppe eingerichtet, um Empfehlungen für die Allgemeinpsychiatrie zum Umgang mit Risikopatienten zu entwickeln [1]. Ausgangspunkt war die Beobachtung, dass ein größer werdender Anteil der Patienten, die in die forensische Psychiatrie eingewiesen werden, bereits vor Begehen der Anlasstat allgemeinpsychiatrisch behandelt wurde. Unterschiedliche Trends in der forensischen Psychiatrie mit zunehmenden Unterbringungszahlen, längeren Unterbringungen und verzögerten Entlassungen - in der Allgemeinpsychiatrie dagegen mit kürzeren stationären Aufenthalten - haben zu einer lebhaften Diskussion über das Verhältnis zwischen forensischer und allgemeiner Psychiatrie geführt [1-6]. Priebe et al. [7,8] sprechen von „Reinstitutionalisierung“, einer Art Gegenreformation in der psychiatrischen Versorgung: mehr Menschen befinden sich im betreuten Wohnen, mehr werden untergebracht und mehr Menschen sind im Gefängnis.

$\mathrm{Zu}$ früh entlassene Patienten, so das Argument, würden straffällig und in den MRV eingewiesen. Es gebe eine „Verschiebung“ von Patienten von der Allgemeinpsychiatrie in die forensische Psychiatrie. Um diesem Phänomen zu begegnen befürworten einige Autoren: a) längere stationäre Aufenthalte für allgemeinpsychiatrische Patienten, speziell diese mit komorbiden Störungen, b) die allgemeinpsychiatrische Verwendung von strukturierten Instrumenten zur Risikoeinschätzung, wie sie in der forensischen Psychiatrie Verwendung finden, und c) ambulante Behandlungsanordnungen (community treatment orders).

Vier Fragen stellen sich bei der kritischen Überprüfung dieser Überlegungen: Kümmert sich die Allgemeinpsychiatrie heute weniger um junge Menschen mit psychotischen Störungen? Wie kommt es, dass sich mehr Menschen mit schizophrenen Störungen im MRV befinden? Welche
Folgen haben kürzere Aufenthalte in der Allgemeinpsychiatrie? Wie effektiv sind strukturierte Risikoeinschätzung und ambulante Behandlungsauflagen?

Psychiatrische Behandlung in Deutschland und vielen anderen Ländern ist einfacher erreichbar als vor 30 Jahren, psychiatrische Kliniken sind näher am Wohnort, die Bedeutung der Früherkennung und -behandlung psychotischer Störungen ist weithin akzeptiert, der Gang zur Psychiaterin oder zum sozialpsychiatrischen Dienst ist kürzer und zahlreiche neue psychiatrisch-psychotherapeutische Praxen wurden eröffnet. Dort, wo ambulante Behandlungspläne erarbeitet werden, Institutsambulanzen bestehen und aufsuchend gearbeitet wird, kümmert sich die Allgemeinpsychiatrie um die Gruppe von schwer erreichbaren Patienten. Laut WHO-Bericht von 2008 [9] haben die meisten Patienten in Deutschland Zugang zu einer langfristigen aufsuchenden Behandlung (assertive outreach). Die größer werdende Gruppe von Drehtürpatienten und rechtlich Betreuten, höhere Fallzahlen und steigende Unterbringungszahlen in der Allgemeinpsychiatrie $[8,10,11]$ sprechen ebenfalls dagegen, dass sich die allgemeinpsychiatrische Versorgung von einer bestimmten Gruppe von Patienten abgewendet habe.

Für die ansteigende Zahl von MRV-Patienten bieten sich drei mögliche Erklärungen an: a) an Schizophrenie erkrankte Menschen werden häufiger straffällig als früher, b) sie bleiben länger im MRV in Behandlung, und c) die Gerichte sind heute eher geneigt in den MRV einzuweisen.

Gründe b) und c) haben kaum etwas mit der Behandlung in der Allgemeinpsychiatrie zu tun, folgen aber gesellschaftlichen Entwicklungen der letzten 40 Jahre: die Behandlung schizophrener Patienten wird im Zuge der Psychiatriereform und seit der Einführung effektiver Psycho- und Pharmakotherapien optimistischer beurteilt; das mag dazu führen, dass die Gerichte eher in den 
MRV einweisen, in der Hoffnung damit das Beste für den Betroffenen zu erreichen.

Mit einem wachsenden Sicherheitsbedürfnis in der Gesellschaft dürfte es zusammenhängen, wenn die Behandlungszeiten im MRV länger werden. In Deutschland und anderen Ländern Europas sind die Gefängnispopulationen angestiegen [12-14]. Verzögerte Entlassungen aus dem MRV können als sensibler und zeitnaher Indikator dieses Trends gelten, denn die Dauer der Behandlung im MRV wird ja im Unterschied zu Haftstrafen nicht im Voraus festgelegt, sondern hängt von aktuellen Faktoren ab, von den vom Patienten noch ausgehenden Risiken, aber auch vom Zeitgeist und möglicherweise sogar vom Tagesgeschehen, wenn sich spektakuläre Verbrechen ereignet haben und die öffentliche Wahrnehmung solcher Ereignisse durch multiple Berichterstattung verstärkt wird.

Möglicherweise werden Menschen, die an Schizophrenie erkrankt sind, heute häufiger straffällig als früher, allerdings scheint dieser Trend im Verhältnis zur Häufigkeit von schweren Gewalttaten insgesamt eher schwach. Bei Tötungsdelikten wurde der Frage in Großbritannien, Neuseeland und Deutschland nachgegangen. Während die Zahl von Tötungsdelikten in England und Wales in den letzten 40 Jahren zugenommen hat, ist der Anteil von Tötungsdelikten, die von psychisch kranken Menschen begangen wurden, rückläufig [15]. In Neuseeland ist der Anteil der von psychisch Kranken begangenen Tötungsdelikte an der Anzahl von Tötungsdelikten insgesamt von 19\% 1970 (vor der Deinstitutionalisierung) auf 5\% im Jahr 2000 gesunken [16]. Die Autoren schließen, dass die vorliegenden Daten keine Rechtfertigung für eine „Reinstitutionalisierung“ darstellen. Erb et al. [17] fanden in Deutschland beim Vergleich einer Kohorte von 1955-1964 mit einer Kohorte von 1992-1996 keinen Anstieg von Tötungsdelikten schizophren erkrankter Menschen seit der Psychiatriereform.

Diese Entwicklung ist auch deshalb bemerkenswert, weil psychotische Störungen heute eher häufiger und früher diagnostiziert werden [18]. Ob dabei die sozial härteren Bedingungen in globalisierten urbanen Siedlungsräumen, Drogenkonsum oder eine größere Bereitschaft zur Diagnosestellung eine Rolle spielen, kann hier offen bleiben, jedenfalls wäre bei häufiger oder früher diagnostizierten psychotischen Störungen eine relative Zunahme von Gewalttaten von Patienten mit solchen Störungen zu erwarten. Dass diese Entwicklung ausgeblieben ist, mag indes mit der Arbeit der Allgemeinpsychiatrie zu tun haben, wenn früher nicht behandelte Patienten heute erreicht und betreut werden.

Kürzere Aufenthaltsdauern in der Allgemeinpsychiatrie wurden als Folge der Psychiatriereform angesehen [4], finden sich jedoch überall in der Medizin aufgrund besserer Behandlungsmöglichkeiten, stationär und ambulant, besseren sozialen Hilfen, geringerem Stigma, weniger Bettenkapazität bei mehr Einweisungen und dem Wissen um die unbefriedigenden Behandlungsergebnisse und unerwünschten Folgen von langen stationären Behandlungen, z.B. die Probleme mit im Allgemeinkrankenhaus erworbenen Infektionen.

In einem Cochrane Review von 2008 [19] wurde untersucht, ob kürzere Aufenthaltsdauern negative Folgen haben. Die Autoren schließen, dass geplante kurze stationäre Aufenthalte bei Patienten mit schweren psychischen Störungen nicht zum sogenannten Drehtürphänomen und zu Behandlungsabbrüchen führen. Kurze Aufenthalte verbessern die Chancen bei der Arbeitssuche [19].
Diskutiert wird, ob sich die Allgemeinpsychiatrie für bestimmte Patientengruppen mehr forensisch-psychiatrischer Arbeitsweisen bedienen sollte: längere Unterbringung, detailliertere Risikoeinschätzung und längerfristige neuroleptische Behandlung, oft gegen den Willen des Patienten. Dabei ist hinsichtlich der späteren Straffälligkeit lediglich die ambulante Zwangsbehandlung einigermaßen gut erforscht: „beim gegenwärtigen Forschungsstand dürften ambulante Behandlungsanordnungen (community treatment orders) keine effektive Alternative zur Standardbehandlung sein. ... Es ist schwer, sich eine andere gesellschaftliche Gruppe vorzustellen, bei der 85 Personen ambulant zwangsbehandelt werden, um eine stationäre Aufnahme zu vermeiden und 238 Personen ambulant zwangsbehandelt werden, um eine Verhaftung zu vermeiden“ (aus einem Cochrane Review von 2005) [20].

Die Frage des prädiktiven Werts bei der Risikoeinschätzung von Patienten mit schizophrenen Störungen wurde von Szmukler diskutiert [21,22]. Weil sich die Risikoeinschätzung auf recht seltene Ereignisse (schwere Gewaltanwendung gegen andere) bezieht, ist bei der Sensitivität und Spezifität derzeitiger Instrumente zu erwarten, dass a) wegen der Seltenheit der Ereignisse viele Patienten als gewalttätig eingeschätzt werden, aber nie gewalttätig werden und deshalb zu Unrecht zwangsbehandelt und untergebracht werden, und dass b) nur ein geringer Anteil der Patienten erfasst wird, die tatsächlich gewalttätig werden. In anderen Worten: jährlich müssten Tausende Patienten zusätzlich untergebracht werden, um ein Tötungsdelikt zu verhindern, und doch würde von den Patienten, die tatsächlich gewalttätig werden, nur einer von acht untergebracht. Die methodischen Schwierigkeiten bei der Kriminalprognose wurden von Volckart [23] ausführlich diskutiert.

Die Folgen konsequenter Anwendung von Risikoeinschätzungsinstrumenten in der allgemeinen Psychiatrie wären zum einen durch präventives Einsperren eine vielfache Verletzung von Menschenrechten [24], denn nur die allerwenigsten der präventiv eingesperrten Personen wären tatsächlich straffällig geworden. Es würde sich an der Häufigkeit von Gewalttaten psychisch Kranker kaum etwas ändern, denn die meisten späteren Gewalttäter würden durch die Risikoeinschätzung nicht erfasst. Zum anderen wäre ein Wettbewerb um begrenzte finanzielle und personelle Ressourcen in der Psychiatrie unausweichlich. Es käme zu einer Umverteilung von Behandlungs- und Betreuungskapazitäten „zugunsten“ solcher Patienten, die als Risikopatienten eingestuft werden, und zuungunsten der Patienten, bei denen aufgrund klinischer Symptome und subjektivem Leiden eine Behandlung geboten ist.

Die behauptete „Verschiebung“ von Patienten von der allgemeinen zur forensischen Psychiatrie aufgrund kürzerer stationärer Behandlungen erscheint wenig plausibel. Die beschriebenen Trends im MRV sind besser erklärbar als Abbildungen eines gesellschaftlichen Trends mit größerem Sicherheitsbewusstsein und geringerer Risikotoleranz. Sollten sich die Empfehlungen zu strukturierter Risikoeinschätzung und ambulanter Zwangsbehandlung in der Allgemeinpsychiatrie durchsetzen, hätte das empfindliche Folgen für die Menschenrechtssituation in der Psychiatrie; begrenzte finanzielle und personelle Mittel würden einer bedarfsgerechten Versorgung dadurch verloren gehen. 


\section{Literatur}

1 Dönisch-Seidel U, van Treek B, Geelen A et al. Zur Vernetzung von forensischer und Allgemeinpsychiatrie. Recht \& Psychiatrie 2007; 25: 184-188

2 Hodgins S, Müller-Isberner R. Preventing crime by people with schizophrenic disorders: the role of psychiatric services. Brit J Psychiatry 2004; 185 : 245-250

3 Munk-Jorgensen P. Has deinstitutionalisation gone too far? Eur Arch Psychiatry Clin Neurosci 1999; 249: 136-143

4 Schanda H, Stompe T, Ortwein-Swoboda G. Psychisch Kranke zwischen Psychiatriereform und Justiz - die Zukunft des österreichischen Maßnahmevollzugs nach $\S 21$ / 1 StGB. Neuropsychiatrie 2006; 20: 40-49

5 Swartz MS, Swanson JW, Wagner HR et al. Assessment of four stakeholder groups' preferences concerning outpatient commitment for persons with schizophrenia. Am J Psychiatry 2003; 160: 1139-1146

6 Zinkler M. Früher entlassen - schneller im Maßregelvollzug? Zum Verhältnis von allgemeiner und forensischer Psychiatrie. Recht \& Psychiatrie 2008; 26: 102-105

7 Priebe S, Badesconyi A, Fioritti A et al. Reinstitutionalisation in mental health care: comparison of data on service provision from six European countries. BMJ 2005; 330: 123-126

8 Priebe S, Frottier P, Gaddini A et al. Mental Health Care Institutions in Nine European Countries, 2002 to 2006. Psychiatr Serv 2008; 59: 570-573

9 World Health Organization. Policies and Practices for mental health in Europe - meeting the challenges. WHO, 2008; www.euro.who.int/ eprise/main/WHO/Progs/MNH/baseline/20080602_1?language=; Aufgerufen am 09.01.2009

10 Haebler D von, Beuscher H, Fähndrich E et al. Wie offen kann die Psychiatrie sein? Dtsch Ärztebl 2007; 104: 1232-1236

11 Hübner-Liebermann B, Hajak G, Spießl H. Versorgungsepidemiologie: Entwicklung in der stationär-psychiatrischen Versorgung 1996-2006. Psychiat Prax 2008; 35: 387-391

12 Sack F. Gewalttätige Jugend - Schlüssel zur Pathologie der Gesellschaft? Schweizerischer Nationalfonds, 1999; NFPNR 40; www.nfp40. ch/pdf/bulletin4.pdf; aufgerufen am 09.01.2009
13 Dünkel F. Der deutsche Strafvollzug im internationalen Vergleich. Universität Greifswald, 2002; www.rsf.uni-greifswald.de/fileadmin/ mediapool/lehrstuehle/duenkel/Strafvollzug_BRD.pdf; aufgerufen am 09.01.2009

14 Council of Europe. Annual Penal Statistics SPACE 1 - Survey 2006. www.coe.int/t/e/legal_affairs/legal_co\%2Doperation/prisons_and_al ternatives/statistics_space_i/List_Space_I.asp\#TopOfPage; aufgerufen am 09.01.2009

15 Taylor PJ, Gunn J. Homicides by people with mental illness: myth and reality. Brit J Psychiatry 1999; 174: 9-14

16 Simpson AIF, Mckenna B, Moskowitz A et al. Homicide and mental illness in New Zealand, 1970-2000. Brit J Psychiatry 2004; 185: 394 398

17 Erb M, Hodgins S, Freese $R$ et al. Homicide and schizophrenia: maybe treatment does have a preventive effect. Criminal Behaviour and Mental Health 2001; 11: 6-26

18 Ajdacic-Gross V, Lauber C, Warnke I et al. Changing incidence of psychotic disorders among the young in Zurich. Schizophrenia Research 2007; 95: 9-18

19 Alwan NA, Johnstone P, Zolese G. Length of hospitalisation for people with severe mental illness. Cochrane Database of Systematic Reviews, 2008 Issue 4

20 Kisely S, Campbell LA, Preston N. Compulsory community and involuntary outpatient treatment for people with severe mental disorders. Cochrane Database of Systematic Reviews, 2005; Issue 3

21 Szmukler G. Homicide inquiries - What sense do they make? Psychiatric Bulletin 2000; 24: 6-10

22 Szmukler G. Violence risk prediction in practice. Brit J Psychiatry 2001; 178: 84-85

23 Volckart B. Zur Bedeutung der Basisrate in der Kriminalprognose. Recht \& Psychiatrie 2002; 20: 105-114

24 Council of Europe - Committee of Ministers. Recommendation No. $\operatorname{REC}(2004) 10$ of the Committee of Ministers to member states concerning the protection of the human rights and dignity of persons with mental disorder and its Explanatory Memorandum. Strasbourg: 2004 